\title{
Décadrages Décadrages
}

cinéma, à travers champs Cinéma, à travers champs

26-27| 2014

Drones, cartographie et images automatisées

\section{Une révolution vintage : Les Grandes Ondes (à l'ouest), de Lionel Baier (Suisse/France/Portugal, 2013)}

\section{Sylvain Portmann}

\section{(2) OpenEdition}

1 Journals

\section{Édition électronique}

URL : https://journals.openedition.org/decadrages/754

DOI : $10.4000 /$ decadrages. 754

ISSN : 2297-5977

Éditeur

Association Décadrages

\section{Édition imprimée}

Date de publication : 10 avril 2014

Pagination : 187-194

ISBN : 978-2-9700668-8-0

ISSN : 2235-7823

Référence électronique

Sylvain Portmann, «Une révolution vintage : Les Grandes Ondes (à l'ouest), de Lionel Baier (Suisse/ France/Portugal, 2013) », Décadrages [En ligne], 26-27 | 2014, mis en ligne le 14 décembre 2015, consulté le 03 avril 2022. URL : http://journals.openedition.org/decadrages/754 ; DOI : https://doi.org/ 10.4000/decadrages.754 


\section{Une révolution vintage: Les Grandes Ondes (à l'ouest), de Lionel Baier (Suisse/France/Portugal, 2013)}

\section{Une drôle de révolution}

Le film met en scène une équipe de la Société Suisse de Radiodiffusion (SSR) envoyée au Portugal en avril 1974 afin de témoigner de l'aide suisse apportée sur place. Après plusieurs tentatives de reportages, l'équipe renonce à toute nouvelle recherche visant à illustrer positivement les (pseudos) efforts de la Confédération tant les résultats escomptés sont misérables. Cette équipe improbable, mobilisée pour l'occasion - composée d'une journaliste carriériste (interprétée par Valérie Donzelli), d'un reporter fruste qui perd la mémoire (int. par Michel Vuillermoz) et d'un technicien en pré-retraite (int. par Patrick Lapp), à laquelle il faudra ensuite ajouter un jeune Portugais qui servira d'interprète (int. par Francisco Belard) - sera le témoin de la Révolution des œillets qui avait

$1 \mathrm{http} / / /$ lesgrandesondes.com (dernière consultation le $\mathrm{I}^{\mathrm{er}}$ juin 20r4). Les critiques sont organisées par lieu de diffusion (France / Suisse alémanique / Suisse romande) et par date. On y trouve même une vidéo clôturant le journal télévisé de 20 h de la chaîne privée TF1 lors de la sortie du film en France.

2 Le premier étant Comme des voleurs (à l'Est) (Suisse, 2006). A propos de ce film, voir Laura Legast, «Mon identité polonaise: Comme des voleurs (à l'Est) de Lionel Baier», Décadrages, nº Io, printemps 2007, pp. IIO-II4.

3 Le réalisateur nous renseigne à ce sujet dans le dossier de presse francophone consacré au film: «Les Grandes Ondes (à l'ouest) fait partie d'une tétralogie autour des quatre points cardinaux en Europe. Le projet, c'est de tracer une sorte de cartographie affective des Européens entre eux. Qu'est-ce qui nous unit les uns aux autres au-delà des institutions. Il y aura donc, je l'espère un (au nord) en Ecosse et un (au sud) en Italie (sic). La situation politique décidera s'il s'agira de comédies, de drames ou de documentaires ...»(Dossier de presse, p. 4). 
4 Les Grandes ondes renvoient de façon imagée aux émissions longue distance (rappelons les trois principales ondes radio: courtes, moyennes et longues), mais également aux grands remous politiques (aux «vagues») des années I970. Enfin à l'ouest: c'est littéralement à l'ouest de l'Europe, au Portugal, que l'histoire se déroule. De façon plus métaphorique l'équipe de radio n'est pas vraiment à son affaire (à l'heure de la révolution, l'équipe suisse ne sait rien des événements), elle est «à l'ouest». mis fin à la dictature (L'Estado Nuovo) instaurée par Antonio de Oliveira Salazar dès 1933. Cet événement majeur de l'Histoire portugaise, mais également européenne, aurait pu paraître traité de façon superficielle, si l'humour corrosif du scénario ne venait régulièrement troubler les images léchées de la photographie et de la mise en scène. Le titre du film ${ }^{4}$ semble à lui seul mettre en place un jeu auquel participent l'Histoire, l'humour et l'esthétique vintage. On y parle de révolution sous une apparence de comédie et avec une forte nostalgie des années I970. C’est donc avec Swissair ou en combi VW qu'on se rend au Portugal, munis d'enregistreurs à bande Nagra et avec des costumes funky (il s'agit d'un film en costumes même si l'époque n'est pas si éloignée) (fig. I-4). Il y a donc une dimension esthétique très artificielle, qui va parfois à l'encontre d'une certaine idée de la mise en scène d'un événement historique rarement revisité de façon comique.

Evoquons un autre traitement du même événement, le film Capitaines d'Avril (Capitães de Abril, Maria de Medeiros, Portugal/Espagne/ Italie/France, 2000) dont l'action se déroule durant vingt-quatre heures, le temps de la révolution. Les événements sont vus à travers le point de vue de deux capitaines portugais insurgés et de l'épouse de l'un d'eux (qui
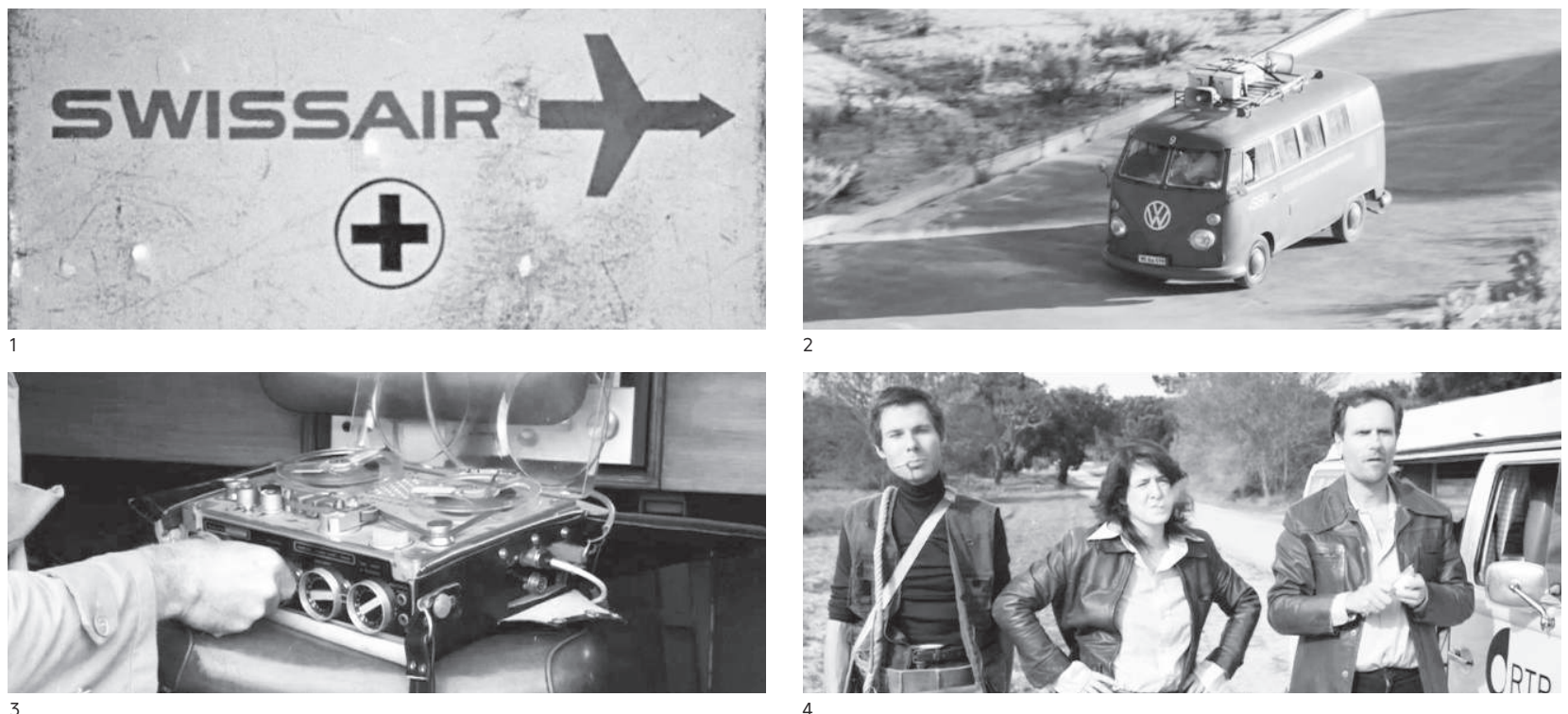
fut l'amante de l'autre: il s'agit d'un triangle amoureux), durant la nuit du 25 avril I974. A la fiction historique se greffe une composante romanesque pesante ${ }^{5}$, mais des aspects concrets sur le plan politique sont inscrits dans le film, comme un discours sur la passation de pouvoir du premier ministre Marcelo Caetano (successeur de Salazar) au général Spinola (qui marque la chute de la dictature) et la nuance que ce geste semble apporter par rapport à l'optimisme que la révolution avait provoqué. En effet, l'écartement rapide des militaires (au profit du général Spinola) qui avaient mené et dirigé les opérations de négociations signalait - c'est en tout cas ce que la mise en scène suggère - la distance que les révolutionnaires avaient déjà pris avec le nouveau pouvoir. Signalons enfin à titre de comparaison la première image du film de Medeiros ( fig. 5) et celle du film de Baier (fig. 6). Les deux plans sont tous deux composés d'images statiques. L'une est en noir et blanc et l'autre en couleur teintée de sépia. L'image en noir et blanc (c'est la première d'une série de dix courtes séquences) est un fragment de film documentaire (caméra à l'épaule tremblant légèrement) montrant les corps mutilés de plusieurs cadavres jonchant le sol. Aucune indication n'est donnée sur sa provenance et il n’y a ni sous-titre ni commentaire audio. Seul son inscription dans le film
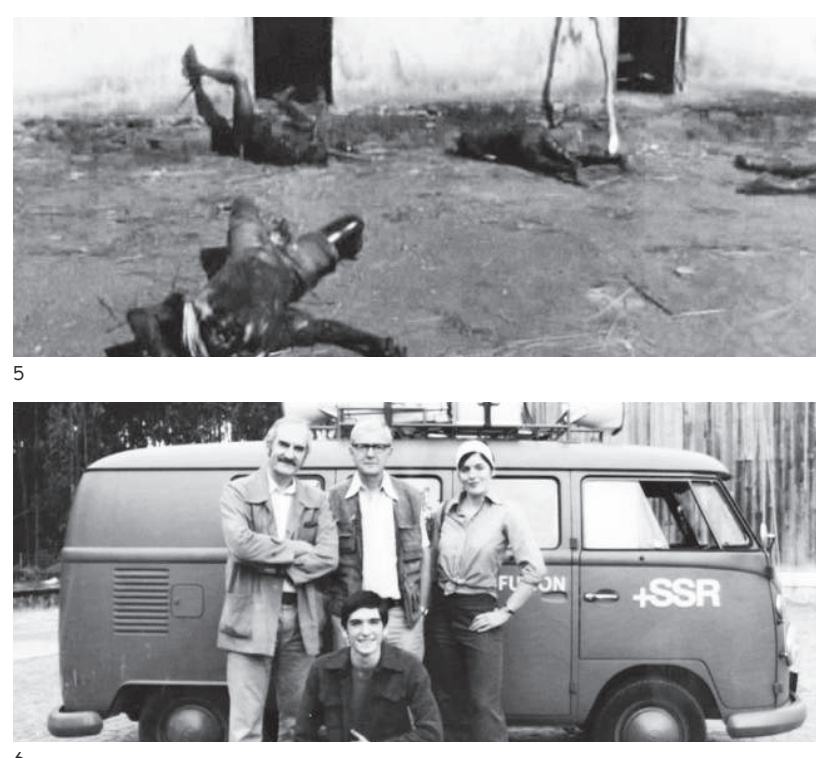

5 Le film de Baier est moins explicite lorsqu'il aborde la libération sexuelle associée à la révolution (vu le ton libertaire des Grandes Ondes, on pourrait penser à une simple proposition scénaristique ou de mise en scène). A ce sujet, voir l'article de Fernando Peixeiro «Révolution des $\mathbb{E}$ Eillets. La révolution érotique», Courrier international (version en ligne), www.courrierinternational. com/article/2014/04/24/la-revolutionerotique, publié le 24 avril 20I4, dernière consultation le $\mathrm{I}^{\text {er }}$ juin $20 \mathrm{O} 4$. 
6 Le film de Ford renvoie à la manière dont on écrit l'Histoire dans (et non à) l'Ouest: la préférence du mythe au fait, à des fins tant identitaires que constitutives du passé (proche) pour les NordAméricains. peut nous faire penser qu'il s'agit là d'une image d'archive, d'actualités, et qu'elle a très certainement été prise en Angola (ou au Mozambique). Sans questionner sa propre authenticité, elle impose un ancrage réaliste mêlé de révolte. Notons que la Révolution des œillets visait également à mettre fin aux guerres coloniales sanglantes menées par le gouvernement portugais - et auxquelles tous les Portugais étaient alors convoqués. Chez Baier, il s'agit d'une photographie qui n'a rien d'«authentique» et qui ne prétend pas l'être. La caméra procède à un zoom avant tandis qu'une voix over dit en portugais (sur un fond sonore composé de chants de grillons):

La mémoire, camarade, c'est comme une nuit d'été. Les milliers d'étoiles sont comme des souvenirs qui scintillent, et qui éclairent la nuit. Chacun raconte une histoire aussi vraie que chaque photo sa légende.

On l'aura compris, le rapport au passé n'est pas le même et la mise en place du second film procède de façon très différente. Il s'agit cette fois d'instiller une ambiance agréable et nostalgique où différents types de discours semblent être mis au même niveau. Qu'il s'agisse de la «mémoire», des «souvenirs», des témoignages des personnages qui se tiennent devant le minibus, de celui qui parle (la fin du film nous apprendra que cette voix est celle de Pelé «aujourd'hui») ou encore de la lecture que chacun peut faire de cette photographie, voire de n'importe laquelle. Comme des voleurs citait une phrase célèbre tirée de The Man Who Shot Liberty Valance (John Ford, E.-U., I962) à un de ses personnages (polonais cette fois): «Lorsque la légende devient la réalité, imprimez la légende [When the legend becomes fact, print the legend]». Baier semble ici faire lointainement écho à Ford, via un de ses précédents films, en proposant une conception mythologique de l'Histoire ${ }^{6}$, bien que le propos du film ne s'y prête pas. S’il convoque des éléments pop en vue de charger positivement ce rapport au passé, aux années I970, cette valorisation artificielle respecte néanmoins une certaine rigueur historique. Peut-être s'agit-il d’une forme de message délivré au spectateur, le prévenant de la nature véritable de l'aventure qui s'apprête à être racontée. Car non seulement celui «qui raconte» le film nous prévient que sa version des faits est donnée comme subjective, mais on apprendra encore par la suite qu'un des personnages principaux perd la mémoire, et que même s'il tente de la fixer sur un support durable (la bande magnétique), le temps qu'il met à le faire lui rend la tâche difficile. 


\section{Du cinéma et des ondes}

Abordant l'Histoire par le biais du registre de la comédie, le film appartient également à un ensemble thématique qu’on pourrait appeler le «film de radio». Car il existe toute une série de films (de fiction pour la plupart) dont l'action se déroule en lien avec une chaîne de radio. On y observe généralement un certain usage de la radio, rattaché soit au divertissement - allant du radio talk show aux émissions du type confessions intimes - soit à l'Information. La mise en scène s'attelle ainsi dans les grandes lignes à présenter dans le premier cas un personnage cloîtré dans les locaux d'une radio, à l'écoute des auditeurs, et dans l'autre à suivre la figure d'un

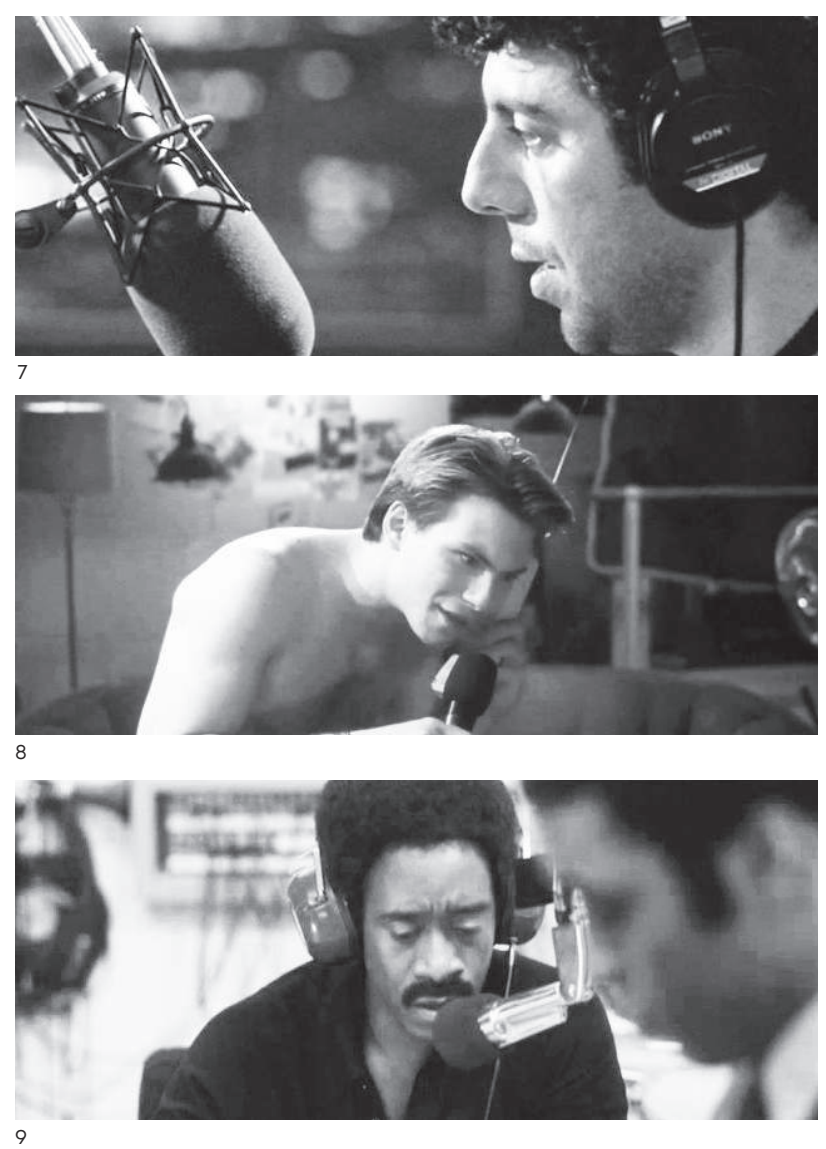


7 Notons que le personnage de Lionel Baier (interprété par Lionel Baier) dans Comme des voleurs est précisément journaliste au département de l'information de la Radio Suisse romande.

8 Autre exemple du même «type», et également une comédie, le film Envoyés très spéciaux (Frédéric Auburtin, France, 2009) qui suit les aventures d'une équipe de radio française censée aller couvrir sur place des événements violents en Syrie. Les deux personnages sont interprétés par Gérard Lanvin (reporter viril) et Gérard Jugnot (technicien naïf) et sont contraints de couvrir l'actualité syrienne depuis le quartier parisien de Barbès à cause de la maladresse du technicien et de l'entêtement orgueilleux du reporter (ils prétendent être sur place alors qu'ils ne se trouvent qu'à quelques kilomètres des studios).

9 Durant la nuit de la révolution, tandis que Cauvin devient un orateur révolutionnaire que tous écoutent, le reste de l'équipe s'adonne à une «partouze». La fin du film les verra récompensés par une distinction radiophonique, «le meilleur reportage francophone de l'année 74", délivré au ministère de la Culture française pour leur reportage au Portugal.

10 Pour des information détaillées à ce sujet, renvoyons à Claude Collin, Ondes de choc: de l'usage de la radio en temps de lutte, Paris/Grenoble, L'Harmattan/Publications de l'Université des langues et des lettres, ig82. reporter aux prises avec l'actualité (souvent lointaine et réservant son lot d'aventures). D'un côté, c'est une certaine conception de «la voix» (ou de l'oreille) dialoguant avec les auditeurs qui est mise en avant, que ce soit pour écouter leurs soucis (comme par exemple Talk Radio (Oliver Stone, E.-U., I988)) (fig. 7) ou pour les pousser à la révolte (qu'elle soit agressive avec Pump up the Volume (Allan Moyle, E.-U., I990) (fig. 8) ou pacifiste avec Talk to Me (Kasi Lemmons, E.-U., 2007)) (fig. 9); et de l'autre, c'est l'information ${ }^{7}$ (nouvelles, culture, etc.). Le film de Baier opte pour la radio en tant que médium d'information ${ }^{8}$, et ceci malgré le fait que les protagonistes répondent tous à des stéréotypes (journaliste baroudeur, animatrice féministe ou technicien maniaque) et que le sérieux exigé par leurs fonctions manque radicalement - ce qui ne pose finalement pas de problème, puisque tous parviennent grâce à leurs drôles de singularités à un résultat "positif» ${ }^{9}$. Ajoutons à cela que ce qui avait déclenché la Révolution des œillets avait précisément été la diffusion par une radio (Rádio Renascença) ${ }^{10}$ de la chanson «Grandôla, Vila Morena» (de Zeca Afonsa), alors interdite par le régime Salazariste.

\section{Le cinéma dans le film}

Autre media d'importance abordé dans le film: le cinéma. Bien qu’il n'y soit présent qu’à travers deux personnages (l'un présent, Pelé, l'autre absent, Marcel Pagnol), il thématise l'accès à un univers culturel que les protagonistes partagent. Pelé, le traducteur portugais du film, raconte qu'il a appris à parler français grâce aux films de Pagnol. Le dossier de presse du film nous apprend que le comédien interprétant son personnage, Francisco Belard, a pour sa part appris la langue via les films de Jacques Tati. Il semblerait donc que l'accent de Raimu ait été préféré à celui de Tati, l'accent méridional recelant peut-être un ressort à la fois poétique - la poésie de Pagnol - et comique - les accents «superposés» formant comme autant de couches, qui nous informent à la fois sur l'origine du personnage (portugaise) et sur son apprentissage de la langue (via les films). C’est donc une certaine culture cinématographique qui permet aux personnage d'avancer dans l'intrigue: les films de Pagnol s'étant propagés hors de France, Pelé a pu apprendre le français, qu’il emploie finalement afin de permettre à des Suisses (romands) de communiquer avec leurs voisins européens. 


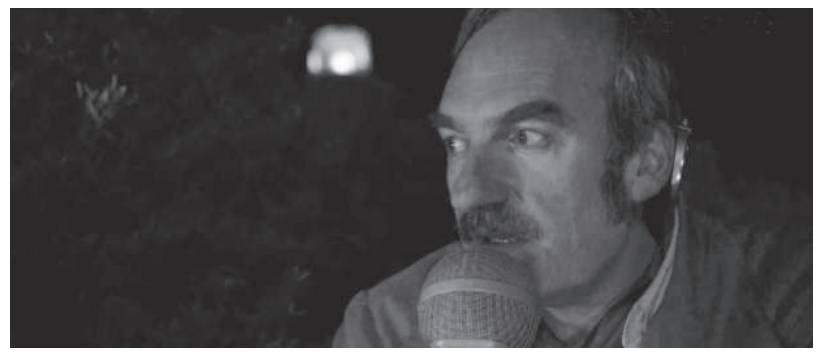

10

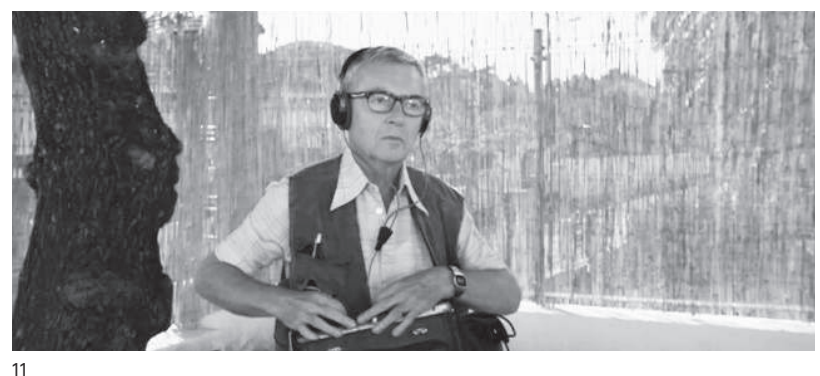

\section{La mémoire par la bande}

Le début du film le soulignait: l'Histoire est intimement liée à la mémoire des personnages. C'est bien Pelé qui est l'énonciateur du film (il se souvient des événements racontés) mais c'est le couple Julie/Cauvin qui se partage la figure du journaliste/reporter véritablement en charge de l'information. Au sein de ce couple, Cauvin occupe la place d'un personnage en proie à des pertes de mémoire - c'est ce qui, malgré son comportement machiste, le rend sympathique. Il enregistre donc sur bande Nagra les événements de la journée (les noms des personnes qu'il rencontre, les lieux, etc.) ( fig. Io) au même titre que le technicien enregistre les reportages (mais en direct) sur le sien (fig. II). Il existe donc un rapport à la fois personnel et professionnel (ou neutre) à l'enregistrement des sons en fonction de leur utilisation. Les deux tendent pourtant à se confondre puisque les aventures rocambolesques des personnages s'entremêlent de façon très serrée avec les événements historiques dont ils sont les témoins. Revenons sur la nuit de la révolution où Cauvin est écouté religieusement par une assemblée de Portugais médusés ( fig. I2I3). Nuit durant laquelle il baragouine un portugais incompréhensible, qu'il pense maîtriser, mais qui pour une fois trouve sa cible. En témoigne cet échange de paroles entre deux auditeurs parmi d’autres: 


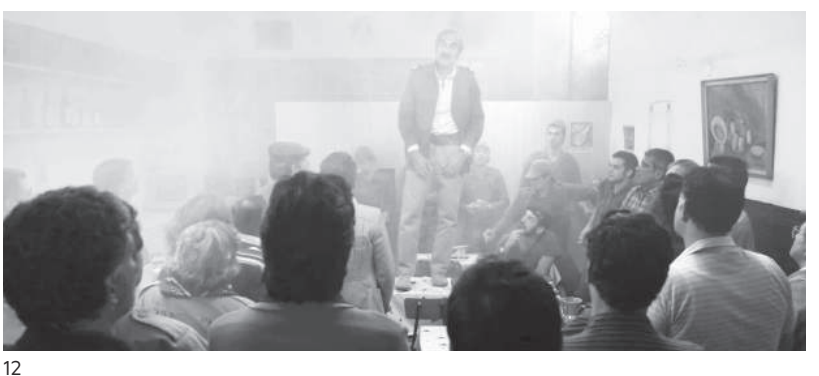

- De quoi il parle?

- Je ne sais pas, mais c'est super.

Le charisme du personnage, associé à la dimension poétique du charabia pseudo-politico-philosophique de son discours, fabrique ainsi une icône révolutionnaire devant nos yeux.

\section{Un clin d'œil au Portugal}

Les Grandes Ondes revient ainsi sur un épisode fondateur de l'Histoire du Portugal, qu'il est bon d'avoir (re)mis en scène. Pour une raison simple, s'accordant à la mémoire de Cauvin: afin d'éviter l'oubli, il faut agir (d'une manière ou d'une autre). Citons à ce propos la revue Jeune Cinéma dont un article (non recensé dans la revue de presse du site des Grandes Ondes) consacré au festival du film de Namur en 2013 nous apprend que cette révolution est désormais tombée dans l'oubli: «Bien des spectateurs, à Namur, ne savaient pas ce que désigne la Révolution des œillets... Mais le public a applaudi Les Grandes Ondes (à l'ouest) (Suisse), de Lionel Baier. On ressort les personnages d'époque: une féministe flamboyante (l'épatante Valérie Donzelli) et un reporter baroudeur (Michel Vuillermoz), qui a interviewé Castro et reçu un éclat d'obus au Viêt-Nam, sont dépêchés sur place par la Radio suisse romande au Portugal afin d'y effectuer un reportage consensuel, sur le rôle apporté par la Suisse au développement du pays " ". Il n'était donc pas inutile de réaliser un film «grand public» sur le sujet.
11 Nicole Gabriel, «Namur (27 septembre - 4 octobre 2013)», Jeune Cinéma, $\mathrm{n}^{\circ} 356$, décembre 2013, p. $4^{2}$. 